\title{
New apparatus for precise synchronous phase shift measurements in storage rings
}

\author{
Boris Podobedov and Robert Siemann \\ Stanford Linear Accelerator Center, Stanford University, Stanford, California 94309
}

(Received 28 August 1998; published 20 November 1998)

\begin{abstract}
Measuring a synchronous phase shift as a function of beam current is commonly done in accelerator physics to estimate the longitudinal impedance of a storage ring vacuum chamber. This measurement is normally done with RF techniques that do not have enough accuracy to detect small phase shifts typical to the newer storage rings. In this paper we report results from a new method for precise measurement of a synchronous phase shift. Our method involves downmixing from the RF frequency to a $\mathrm{kHz}$ range and then using an audio DSP lock-in amplifier for the actual phase detection. This paper describes the idea and the advantages of a new method as well as its practical implementation in the apparatus we build for precise synchronous phase measurements in the Stanford Linear Collider damping rings. The results of those measurements are also presented. [S1098-4402(98)00020-2]
\end{abstract}

PACS numbers: 07.50.-e, 29.27.Bd

\section{INTRODUCTION}

Measuring a synchronous phase shift as a function of beam current gives the so-called loss factor, which is related to the ring impedance and affects the longitudinal beam dynamics. There are elaborate computational methods to calculate the impedance by summing up the contributions of individual vacuum chamber elements, and the measurement of the loss factor provides a check of the calculations and the vacuum chamber manufacturing and assembly procedure.

Although conceptually simple, the synchronous phase shift measurement can be difficult in practice, especially for the newer storage rings. The loss factor tends to be small, resulting in a total phase shift on the order of a degree or less which is hard to measure accurately with traditional experimental techniques. A similar problem arises when the loss factor is relatively high but measurements are performed at low current to avoid the effect of bunch lengthening or instabilities. In this paper we introduce a new low cost apparatus that offers significantly higher precision and flexibility than commonly used setups. We will also describe our successful use of this apparatus to measure the synchronous phase shift in the Stanford Linear Collider (SLC) damping rings.

\section{PHYSICS OF A SYNCHRONOUS PHASE SHIFT AND PREDICTIONS FOR THE SLC DAMPING RINGS}

The energy balance of the stored beam can be written as

$$
e V_{\mathrm{RF}} \cos \left(\varphi_{0}+\Delta \varphi\right)=U_{0}+k e^{2} N
$$

where $V_{\mathrm{RF}}$ is the accelerating voltage, $\varphi_{0}$ is the zero current synchronous phase, $U_{0}$ is the synchrotron radiation energy loss per turn, $e N$ is the total beam charge, $k$ is the loss factor, and $\Delta \varphi$ is the synchronous phase shift. When the latter is small, we get for the loss factor

$$
k=-\frac{\Delta \varphi}{e N} V_{\mathrm{RF}} \sin \varphi_{0}
$$

For a given longitudinal beam density $\rho(z)$, the loss factor can be related to the wakefield $W(z)$ [total wakefield for the distribution $\rho(z)$ ] or to the real part of the impedance $Z(\omega)$ as [1]

$$
\begin{aligned}
k & =\int_{-\infty}^{\infty} W(z) \rho(z) d z \\
& =\frac{1}{2 \pi} \int_{-\infty}^{\infty} \operatorname{Re}[Z(\omega)]|\tilde{\rho}(\omega)|^{2} d \omega,
\end{aligned}
$$

where $\rho(z)$ is normalized to 1 and $\tilde{\rho}(\omega)$ is the Fourier transform of $\rho(z)$. For short Gaussian bunches, the $\omega$ dependence of $Z$ can be neglected above so that we get for the real part of the impedance

$$
R=\frac{2 \sqrt{\pi} \sigma k}{c},
$$

where $\sigma$ is the rms bunch length and $c$ is the velocity of light. For the SLC damping rings, the longitudinal wakefield $W(z)$ has been calculated numerically [2,3] for a $1 \mathrm{~mm}$ rms Gaussian bunch. For the low current bunch length of $\sigma_{0}=5.3 \mathrm{~mm}$ corresponding to $800 \mathrm{kV}$ nominal RF voltage, this calculation yields the total loss factor of $15 \mathrm{~V} / \mathrm{pC}$ which corresponds to $950 \Omega$ resistive impedance. This loss factor substituted into Eq. (2) where $\varphi_{0}=84^{\circ}$ calls for the phase shift of $1.7^{\circ}$ per $10^{10}$ particles. This is the value we have to be able to measure.

\section{TRADITIONAL WAY TO MEASURE THE SYNCHRONOUS PHASE SHIFT AND ITS LIMITATIONS}

Although other signals carrying beam phase information exist, the simplest one to use is a beam position monitor (BPM) pickup signal. This signal includes the fundamental harmonic of the RF frequency. The phase shift of this harmonic relative to the RF in the accelerating cavity defines the synchronous phase up to some constant offset. 
For large phase shifts the synchronous phase can be measured simply with an oscilloscope or a mixer-based phase detector. Smaller phase shifts require more accurate tools. The most precise, general purpose instrument to measure the phase of RF signals is the vector voltmeter. It is traditionally the instrument of choice for synchronous phase shift measurements $[4,5,6]$.

However, as we found in our experiments, we could not measure the synchronous phase shift reliably with a Hewlett-Packard (HP) 8508A vector voltmeter. This is not surprising because the accuracy that standard vector voltmeters provide is not sufficient for the SLC damping rings. For example, the specifications for the HP 8508A state a $0.8^{\circ}$ phase accuracy within a $1 \mathrm{mV}$ to $100 \mathrm{mV}$ input signal range and a several times lower accuracy outside of it [7]. Another disadvantage of this device is the lack of convenient control over the averaging constant which is important for synchronous phase measurement. Indeed, in a noisy environment a significant increase in sensitivity can be achieved from averaging the phase over the interval comparable to the sampling time.

To overcome these limitations of the vector voltmeter we came up with the following solution.

\section{NEW APPARATUS}

In our apparatus we modify the approach described above in two ways. First, we do not perform the phase comparison at the fundamental RF frequency but, rather, downmix both beam and RF cavity signals to roughly $25 \mathrm{kHz}$ where audio measurement techniques are available. Second, for the actual phase measurement we use the SR830 DSP lock-in amplifier manufactured by Stanford Research Systems. This device has a relative phase error of $0.01^{\circ}$, adequate input sensitivity and temperature stability, and provides complete control over the time averaging constant. Other features we found useful for this measurement include power line notch filters in the signal arm, auxiliary ADC channels, and complete GPIB capability.

The basic idea of our apparatus is shown in Fig. 1. To provide an LO arm for the downmixing, we use an RF synthesizer. The low-pass filters cut out the higher frequency mixing products. Since a bridge is implemented, synthesizer frequency drift should not, in the first order, result in phase error. Note that the total cost of this setup is a fraction of that for the vector voltmeter. Also, the system is rather flexible. Indeed, to switch to another RF frequency one needs to either reprogram the synthesizer or replace it when the frequency is substantially different. Additionally, this setup can be used in other experiments that require phase measurement of RF signals of practically any frequency. For example, a similar approach was used in a homemade $W$-band vector network analyzer where the phase and the amplitude of a $92 \mathrm{GHz}$ signal were measured [8]. Finally, filtering a beam signal at the fundamental harmonic can be hard for larger rings where a very high- $Q$ RF filter may be needed. However, with this setup we can easily insert one more stage of downmixing to some common frequency where appropriate off-the-shelf crystal filters are available.

\section{EXPERIMENTAL DETAILS}

In order to accurately measure the phase it is not enough to have a precise lock-in amplifier. Care has to be taken about other elements of the system as well as the machine conditions. Here we summarize our experience from the SLC measurements.

Input cables. The typical electrical length change in cables runs from $150 \mathrm{PPM} /{ }^{\circ} \mathrm{C}(\mathrm{RG} 223)$ to $\sim 6 \mathrm{PPM} /{ }^{\circ} \mathrm{C}$ (HELIAX). Therefore, a temperature drift during a measurement can significantly alter the result if the cable runs are long. In the SLC ( $100 \mathrm{ft}$ of RG223), we found that using a temperature stabilized cable significantly improves measurement reproducibility. Unfortunately, this cable was available only for part of the distance needed and the temperature in the room with our setup was not fully controlled. This may explain our experience of better reproducibility when the measurements were taken at night so that the outside temperature did not change much.

$B P M$ signal filter. To measure the phase correctly, the input signal to the lock-in amplifier should not have significant amplitude modulation. Approximating a filter as an oscillator of a quality factor $Q$ gives a requirement $Q / h \geq 1$, where $h$ is the harmonic number. For the SLC damping rings $\left(f_{\mathrm{RF}}=714 \mathrm{MHz}, h=84\right)$, we made a simple coaxial cavity filter with $Q \approx 300$.

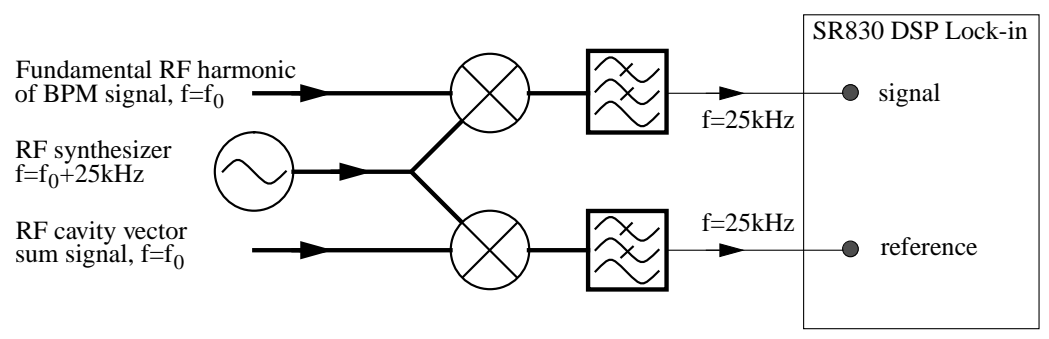

FIG. 1. The idea of the apparatus. 


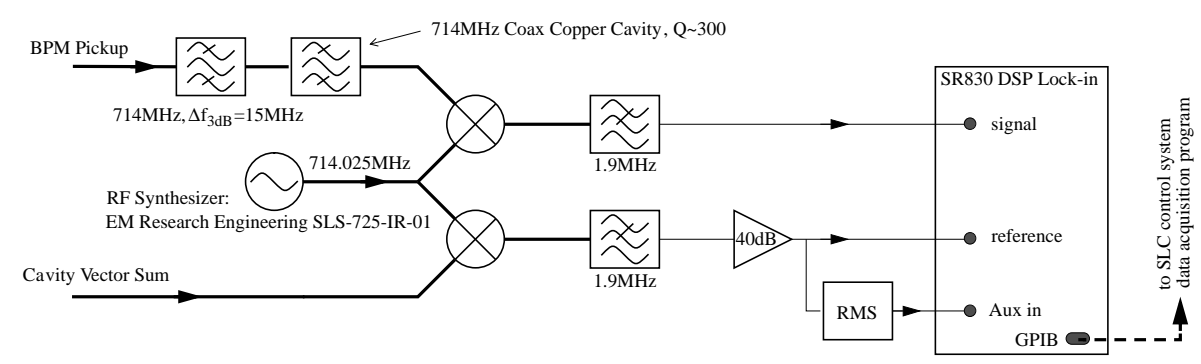

FIG. 2. The setup for synchronous phase measurement in the SLC damping rings.

Vector sum. Equation (1) includes the total accelerating voltage. To measure it the pickup voltages from the individual cells must be summed vectorially. This signal is available for the SLC since it is used in the so-called direct feedback loop [9].

Signal levels. The SR830 specifications require at least $200 \mathrm{mV}_{\text {peak-peak }}$ (into $1 \mathrm{M} \Omega$ ) reference arm input signal. We had to use an audio amplifier to bring the reference signal above this level. To ensure that the amplitude stayed constant we monitored it by a homemade rms detector with the output connected to one of the auxiliary inputs of SR830 (Fig. 2). A related issue here is the isolation of the power splitter that divides the LO signal. We had to make sure that it is high enough so that the cavity signal does not leak through the coupler and does not significantly distort the beam signal or vice versa.

Beam lifetime and the lock-in averaging constant. To get a significant current decay, the store should be comparable to the beam lifetime. There are simple ways of shortening the lifetime (with scrapers, transverse detuning, etc.) to accelerate the measurement and reduce the temperature drift effect. However, the decay has to be rather smooth because rapid drops in the input signal to the lock-in amplifier may result in phase measurement error. In the SLC (lifetime on the order of $10 \mathrm{~min}$ and no scraper is available to reduce it), we tried the transverse detuning but could not make it smooth enough. We proceeded with our measurements using nominal lifetime stores. The data were sampled about every $3 \mathrm{~s}$, and the lock-in time averaging constant was set to $1 \mathrm{~s}$. Shorter settings resulted in noisier data but did not lead to a different phase slope with the current.

Feedbacks. If the vector sum signal is properly derived, most feedback loops should not affect the measurement. This, however, does not hold true for two SLC feedback loops. One of them is the so-called $S$-band feedback that adjusts the beam phase for subsequent injection to the linac. The other is the direct feedback loop that effectively changes the cavity shunt impedance. These two feedbacks had to be turned off for our measurements.

\section{DAMPING RING MEASUREMENT RESULTS}

The measurements we describe here were taken in the positron damping ring. Similar results were later obtained in the other (electron) damping ring that has an almost identical vacuum chamber. The setup we used is shown schematically in Fig. 2. Single beam stores were performed at different values of accelerating voltage clustered near $600 \mathrm{kV}, 800 \mathrm{kV}$, and $1000 \mathrm{kV}$. The total beam charge was $1-1.5 \times 10^{10}$ particles per bunch at the beginning of each store, which is below the known longitudinal instability threshold. The stores lasted $10-15 \mathrm{~min}$, so that the current decayed roughly by a factor of 2 (Fig. 3a). Since many systematic errors could show up as a linear change of phase with time, which could hide real effects, we took several measurements at each value of accelerating voltage. The total number of stores was 17 .

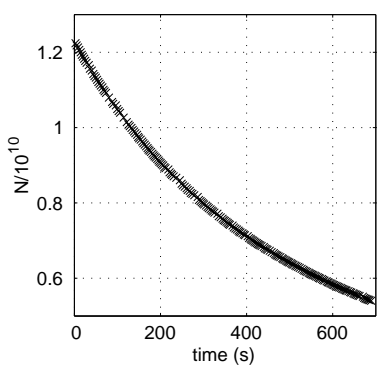

(a)

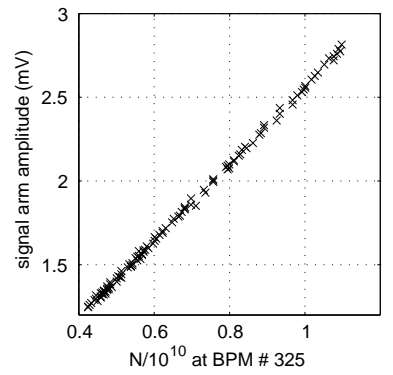

(b)

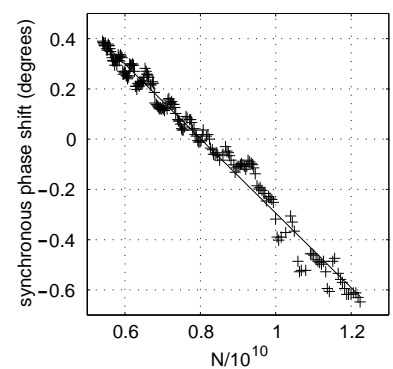

(c)

FIG. 3. Typical data sample: (a) charge stored vs time, (b) lock-in signal amplitude vs BPM, and (c) phase shift vs charge stored (data and linear fit). 


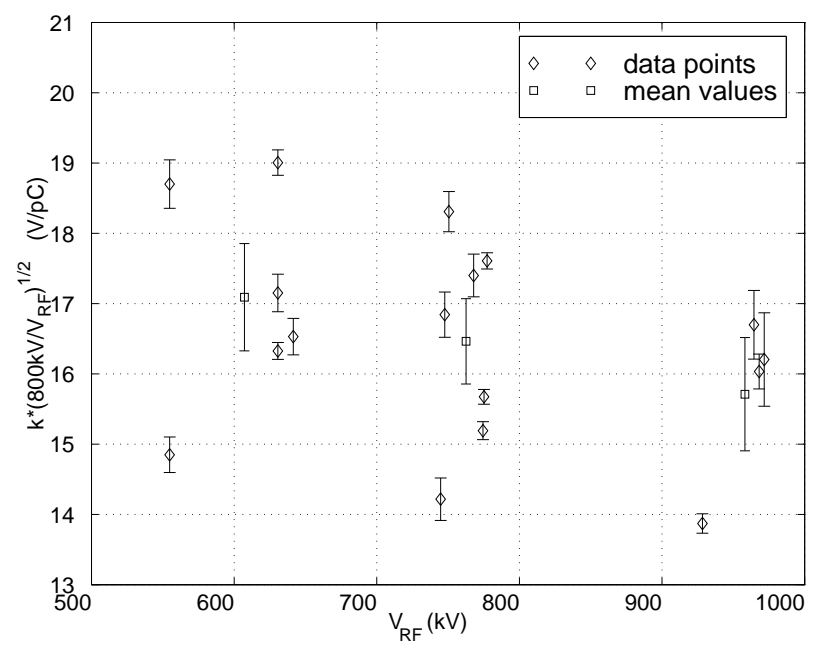

FIG. 4. Scaled loss factor vs accelerating voltage. The results from each store are plotted with the statistical error of the fit (diamonds). Also plotted are the mean values within each group (squares) with their errors derived from the rms spread of the individual stores.

For each store we recorded some of the RF system parameters as well as the beam current; these values are routinely available from the SLC control system. Simultaneously, we sampled five values with the lockin amplifier, namely, the lock status bit, reference arm amplitude, reference arm frequency, signal arm amplitude, and signal arm phase. All but the last were recorded for diagnostic purposes to ensure a solid lock to the external reference with stable frequency and amplitude. The signal arm amplitude was proportional to the beam current (Fig. 3b) which argues that the signal was largely beam induced. The phase shift measured by the lock-in was the actual synchronous phase shift and, as expected, it turned out to be linear with the beam current (Fig. 3c). The phase shift slope with current substituted in Eq. (2) allowed us to extract the value for the loss factor. The results for 17 stores at different RF voltages are shown in Fig. 4. The data are consistent with a constant, justifying the assumptions made in Eq. (4) together with $\sigma^{2} \sim V_{\mathrm{RF}}$ scaling. Using these assumptions we found the average nominal value for the loss factor $k_{0}=16.5 \mathrm{~V} / \mathrm{pC} \pm 15 \%$ which corresponds to about $1 \mathrm{k} \Omega$ resistive component in the longitudinal impedance. This measured value is in good agreement with calculations [2,3]. The error is largely systematic and comes from the uncertainty in absolute values of beam current and accelerating voltage; the error in the mean from the data points in Fig. 4 is less than 3\%. It is also possible to estimate the loss factor for a given accelerating voltage without making any assumptions on bunch length scaling. For example, for $V_{\mathrm{RF}}=760 \mathrm{kV}$ the loss factor $k(760 \mathrm{kV})=16 \mathrm{~V} / \mathrm{pC}$, which is consistent with $k_{0}$.

\section{SUMMARY}

We have designed and built a new apparatus that allows for precise synchronous phase measurement in the storage rings. Our apparatus compares favorably with other techniques due to increased accuracy and flexibility and lower cost. We have successfully used our apparatus in the SLC damping rings where the total phase shifts measured were about $1^{\circ}$. The loss factor value was obtained from our measurement and agrees well with calculations.

\section{ACKNOWLEDGMENTS}

The authors thank Mike Seidel and Dave Whittum for initiating this work, and Al Menegat for help making the filter cavity. This work was supported by the Department of Energy Contract No. DE-AC03-76SF00515.

[1] A. Chao, in Physics of Collective Beam Instabilities in High Energy Accelerators (Wiley, New York, 1993).

[2] K.L.F. Bane and K. Oide, in Proceedings of the 1995 Particle Accelerator Conference, edited by L. T. Gennari (IEEE, Piscataway, NJ, 1996), p. 3150.

[3] K.L.F. Bane and C.-K. Ng, in Proceedings of the 1993 Particle Accelerator Conference, edited by S.T. Corneliussen (IEEE, Piscataway, NJ, 1993), p. 3432.

[4] M. A. Allen et al., IEEE Trans. Nucl. Sci. 22, 1838 (1975).

[5] L. Rivkin et al., IEEE Trans. Nucl. Sci. 32, 2628 (1985).

[6] W. Anders et al., in Proceedings of the XVth International Conference on High Energy Accelerators, 1992, edited by J. Rossbach (World Scientific, Singapore, 1992), p. 1121.

[7] Hewlett-Packard, HP 8508A Vector Voltmeter Operating and Service Manual, 1988.

[8] R.H. Siemann, SLAC Report No. SLAC-PUB-7884, 1998.

[9] M. Minty and R.H. Siemann, Nucl. Instrum. Methods Phys. Res., Sect. A 376, 301 (1996). 